\title{
Automated Filter Parameter Selection using Measures of Noiseness
}

\author{
Ajit Rajwade, Anand Rangarajan and Arunava Banerjee, \\ Department of CISE, University of Florida, Gainesville, USA \\ avr, anand, arunava @ cise.ufl.edu
}

\begin{abstract}
Despite the vast body of literature on image denoising, relatively little work has been done in the area of automatically choosing the filter parameters that yield optimal filter performance. The choice of these parameters is crucial for the performance of any filter. In the literature, some independence-based criteria have been proposed, which measure the degree of independence between the denoised image and the residual image (defined as the difference between the noisy image and the denoised one). We contribute to these criteria and point out an important deficiency inherent in all of them. We also propose a new criterion which quantifies the inherent 'noiseness' of the residual image without referring to the denoised image, starting with the assumption of an additive and i.i.d. noise model, with a loose lower bound on the noise variance. Several empirical results are demonstrated on two well-known algorithms: $N L$-means and total variation, on a database of 13 images at six different noise levels, and for three types of noise distributions.
\end{abstract}

\section{Introduction}

Contemporary literature on image denoising is certainly very vast. Some of the popular approaches include partial differential equations (PDEs) [15], wavelet thresholding [8], learning-based approaches like KSVD [10] and nonlocal approaches [4]. More recently, new approaches based on spatially-varying regressions [20] and spatially varying convolutions [19] have also been developed. An approach to filtering using level curves in a mean-shift framework has been developed in [17].

The typical denoising technique requires tuning parameters that are critical for its optimal performance. In denoising experiments reported in contemporary literature, the filter performance is usually measured by the mean-squared error (MSE) between the denoised and the original image. The parameters are picked so as to yield the least MSE value for a particular filter, but this requires knowledge of the original image and is not extensible to real-world denoising situations. Hence we need criteria for parameter selection that do not refer to the original image. In this paper, we classify these criteria into two types: (1) independence-based criteria that measure the degree of independence between the denoised image and the residual, and (2) criteria that measure how noisy the residual image is, without referring to the denoised image. We contribute to and critique criteria of type (1), and proposed those of type (2). Our criteria make the assumption that the noise is i.i.d. and additive, and that a loose lower-bound on the noise variance is known.

This paper is organized as follows: Section 2 reviews existing literature for filter parameter selection, followed by a description of the proposed criteria in Section 3, experimental results in Section 4 and discussion in Section 5.

\section{Literature Review}

In PDE-based denoising, the choice of stopping time for the PDE evolution is a crucial parameter. Some researchers propose to stop the PDE when the variance of the residual equals the variance of the noise, which is assumed to be known [18], [24]. This method ignores higher order statistics of the noise. Others use a hypothesis test between the empirical distribution of the residual and the true noise distribution [16] for polynomial order selection in regressionbased smoothing. However the exact variance of the noise or its complete distribution is usually not known in practical situations. A decorrelation-based criterion independently proposed in [14] and [2] does not require any knowledge of the noise distribution except that the noise is independent of the original signal. As per this criterion, the optimal filter parameter is chosen to be one which minimizes the correlation coefficient between the denoised and the residual images, regardless of the noise variance. This criterion however has some problems: (1) in the limit of extreme oversmoothing or undersmoothing, the correlation coefficient is undefined as the denoised image could become a constant image, (2) it is too global a criterion (though using a sum of local measures is a ready alternative) and (3) it ignores higher-order dependencies. A solution to the third 
issue is suggested by us in Section 3.

It should be noted that all the aforementioned criteria (as also the ones we suggest in this paper) are necessary but not sufficient for parameter selection. Gilboa et al. [11] attempt to alleviate this by selecting a stopping time that seeks to maximize the signal-to-noise-ratio (SNR) directly. Their method however requires an estimate of the rate of change of the covariance between the residual and the noise w.r.t. the filtering parameter. This estimate in turn requires full knowledge of the noise distribution. Saddled with this method is the assumption that the covariance between the residual for any image and the actual noise, can be estimated from a single noise-image generated from the same noise distribution. This assumption is not justified theoretically though experimental results are impressive (see [11] for more details). Vanhamel et al. [21] propose a criterion that maximizes an estimate of the correlation between the denoised image and the true, underlying image. This estimate, however, can be computed only by using some assumptions that have only experimental justification. In wavelet thresholding methods, risk based criteria have been proposed for the optimal choice of the threshold for the wavelet coefficients. Some of these (such as SURE - Stein's unbiased risk estimator) [8] again require complete knowledge of the noise model, whereas others merely use the number of samples in the image (the size of the unknown signal) for computation of the threshold [7]. Although several asymptotic properties have been proved for these estimates, we are not aware of their small sample-size behavior (which is the practical setting). Recently, Brunet et al. have developed no-reference quality estimates of the MSE between the denoised image and the true underlying image [3]. These estimates do not require knowledge of the original image, but they do require knowledge of the noise variance and obtain a rough, heuristic estimate of the covariance between the residual and the noise. Moreover the performance of these estimates has been tested only on Gaussian noise models.

\section{Theory}

\subsection{Independence measures}

In what follows, we shall denote the denoised image obtained by filtering a noisy image $I$ as $D$, its corresponding residual as $R$ (note that $R=I-D$ ) and the true image underlying $I$ as $J$. As mentioned earlier, independence-based criteria have been developed in image processing literature. In cases where a noisy signal is oversmoothed (locally or globally), the residual image clearly shows the distinct features from the image (referred to as 'method noise' in [4]). This is true even in those cases where the noise is independent of the signal. Independence-based criteria are based on the assumption that when the noisy image is filtered optimally, the residual would contain mostly noise and very little signal and hence it would be independent of the denoised image. It has been experimentally reported in [14] that the absolute correlation coefficient (denoted as $C C$ ) between $D$ and $R$ decreases almost monotonically as the filter smoothing parameter is increased (in discrete steps) from a lower bound to a certain 'optimal' value, after which its value increases steadily until an upper bound. However, CC ignores anything higher than second-order dependencies. To alleviate this problem, we propose to minimize the mutual information (MI) between $D$ and $R$, as a criterion for parameter selection. This has been proposed as a (local) measure of noiseness earlier in [3], but it has been used in that paper only as an indicator of areas in the image where the residual is unfaithful to the noise model, rather than as an explicit parameter-selection criterion. In this paper, we also propose to use the following information-theoretic measures of correlation from [6] (see page 47) as independence criteria:

$$
\begin{gathered}
\eta_{1}(R, D)=1-\frac{H(R \mid D)}{H(D)}=\frac{M I(R, D)}{H(D)} \\
\eta_{2}(R, D)=1-\frac{H(D \mid R)}{H(R)}=\frac{M I(R, D)}{H(R)} .
\end{gathered}
$$

Here $H(X)$ refers to the Shannon entropy of $X$, and $H(X \mid Y)$ refers to the conditional Shannon entropy of $X$ given $Y . \eta_{1}$ and $\eta_{2}$ both have values bounded from 0 (full independence) to 1 (full independence).

A problem with all these criteria (CC,MI, $\left.\eta_{1}, \eta_{2}\right)$ lies in the inherent probabilistic notion of independence itself. In the extreme case of oversmoothing, the 'denoised' image may turn out to have a constant intensity, whereas in the case of extreme undersmoothing (no smoothing or very little smoothing), the residual will be a constant (zero) signal. In such cases, $C C, \eta_{1}, \eta_{2}$ are ill-defined whereas $M I$ turns out to be zero (its least possible value). What this indicates is that these criteria have the innate tendency to favor extreme cases of under- or over-smoothing. In practical applications, one could evade this issue by choosing a local minimum of these measures within a heuristically chosen interval in the parameter landscape from 0 to $\infty$, but we wish to drive home a more fundamental point about the inherent flaw in using independence measures. Moreover, it should be noted that localized versions of these measures (i.e. sum of local independence measures) may produce false optima if the filtering algorithm smoothes out local regions with fine textures at intermediate steps.

\subsection{Characterizing residual 'noiseness'}

Given the fact that the assumed noise model is i.i.d. and signal independent, we expect the residual produced 
by an ideal denoising algorithm to obey these characteristics. Therefore, patches from residual images are expected to have similar distributions if the filtering algorithm has performed well. Our criterion for characterizing the residual 'noiseness' is rooted in the framework of statistical hypothesis testing. We choose the two-sample KolmogorovSmirnov (KS) test to check statistical homogeneity. The two-sample KS test-statistic is defined as

$$
K=\sup _{x}\left|F_{1}(x)-F_{2}(x)\right|
$$

where $F_{1}(x)$ and $F_{2}(x)$ are the respective empirical cumulative distribution functions (ECDF) of the two samples, computed with $N_{1}$ and $N_{2}$ points. Under the null hypothesis when $N_{1} \rightarrow \infty, N_{2} \rightarrow \infty$, the distribution of $K$ tends to the Kolmogorov distribution, and is therefore independent of the underlying true CDFs themselves. Therefore the $K$ value has a special meaning in statistics. For a 'significance level' $\alpha$ (the probability of falsely rejecting the null hypothesis that the two ECDFs were the same), let $K_{\alpha}$ be the statistic value such that $P\left(K \leq K_{\alpha}\right)=1-\alpha$. The null hypothesis is said to be rejected at level $\alpha$ if $\frac{\sqrt{N_{1} N_{2}}}{N_{1}+N_{2}} K>K_{\alpha}$. Given a value of the test-statistic computed empirically from the samples (denoted as $\hat{K})$, we term $P(K \leq \hat{K})$ (under the null-hypothesis) as the p-value.

Most natural images (apart from homogenous textures) show a considerable degree of statistical dissimilarity. To demonstrate this, we performed the following experiment on all 480 images from the Berkeley database [13]. Each image at four scales with successive downsampling factor of $\frac{2}{3}$ was tiled into non-overlapping patches of sizes $s \times s$ where $s \in\{16,24,32\}$. The two-sample KS test for $\alpha=0.05$ was performed for patches from these images. The average rejection rate was $81 \%$ which indicates that different regions from each image have different distributions. It should be noted that the tiling of the image into patches was very important: a KS test between sample subsets from random locations produced very low reject rates. A similar experiment with the same scales and patch sizes run on pure Gaussian noise images resulted in a rejection rate of only $7 \%$ for $\alpha=0.05$. Next, Gaussian noise of $\sigma=0.005$ (for intensity range $[0,1]$ ) was added to each image. Each image was filtered using the Perona-Malik filter [15] for 90 iterations with a step size of 0.05 and edgeness criterion of $\gamma=40$ and the residual images were computed after the last iteration. The KS-test was performed at $\alpha=0.05$ between patch pairs from each residual image. The resulting rejection rate was $41 \%$, indicating strong heterogeneity in the residual values. As structural patterns were clearly visible in all these residual images, we therefore conjecture that statistical heterogeneity is a strong indicator of the presence of structure. Moreover the percentage reject rate (denoted as $h$ ), the average value of the KS-statistic (i.e. $K$ ) and the average negative logarithm of the $\mathrm{p}$-values from each pairwise test (denoted as $P$ ) are all indicators of the 'noiseness' of a residual (the lower the value, the noisier and hence more desirable the residual). Hence these measures act as criteria for filter parameter selection ${ }^{1}$. We prefer the criteria $P$ and $K$ to $h$ because they do not require a significance level to be specified a priori.

The advantage of the KS-based measure over MI or CC is that values of $P$ and $K$ are high in cases of image oversmoothing (as the residual will then contain more and more structure). This is unlike MI or CC which will attain false minima. This is demonstrated in Figure 1 where the decrease in the values of MI or CC at high smoothing levels is quite evident. Just like MI or CC, the KS-based criteria do not require knowledge of the noise distribution or even the exact noise variance. However all these criteria could be fooled by the pathological case of zero or very low denoising. This is because in the very initial stages of denoising (obtained by, say, running a PDE with a very small stepsize for very few iterations), the residual is likely to be devoid of structure and independent of the (under)smoothed image. Consequently, all measures: $\mathrm{MI}, \mathrm{CC}, K$ and $P$ will acquire (falsely) low values. This problem can be avoided by making assumptions of the range of values for the noise variance (or a loose lower-bound), without requiring exact knowledge of the variance. This has been the strategy followed implicitly in contemporary parameter selection experiments (e.g. in [14] the PDE stepsizes are chosen to be 0.1 and 1). In all our experiments, we make similar assumptions. The exception is that KS-based measures do not require any upper bound on the variance to be known: just a lower bound suffices.

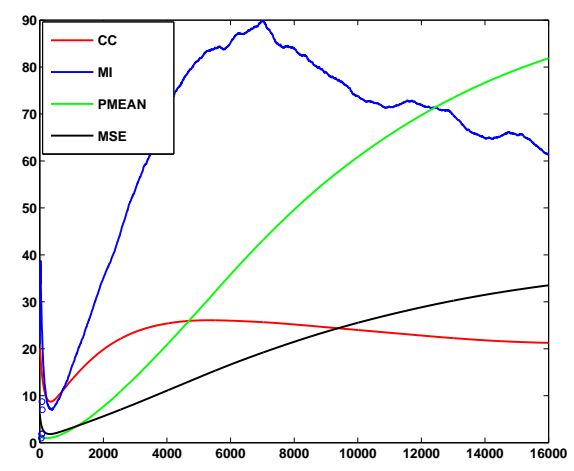

Figure 1. Plots of CC, MI, $P$ and MSE on an image subjected to upto 16000 iterations of total variation denoising.

\footnotetext{
${ }^{1}$ For computing $P$, there is the assumption that the pairwise tests between individual patches are all independent, for the sake of simplicity.
} 


\section{Experimental Results}

To demonstrate the effectiveness of the proposed criteria, we performed experiments on all the 13 images from Lansel's benchmark dataset [1]. All images from the dataset were down-sized from $512 \times 512$ to $256 \times 256$. We experimented with 6 noise levels $\sigma_{n}^{2} \in\left\{10^{-4}, 5 \times 10^{-4}, 10^{-3}, 5 \times\right.$ $\left.10^{-3}, 0.01,0.05\right\}$ on an intensity range of $[0,1]$, and with three additive zero-mean noise distributions: Gaussian (the most commonly used assumption), bounded uniform (noise due to quantization) and negative exponential (noise model used for SAR imagery). The lower-bound assumed on the noise variance was $10^{-6}$ in all experiments. Two filtering algorithms were tested: the non-local means (NL-means) filter [4] and total variation (TV) [18]. The equation for the NL-means filter is as follows:

$$
\begin{array}{r}
\hat{I}(x)=\frac{\sum_{x_{k} \in N(x ; S R)} w_{k}(x) I\left(x_{k}\right)}{\sum_{x_{k} \in N(x ; S R)} w_{k}(x)}, \\
w_{k}(x)=\exp \left(-\frac{\left\|q(x ; Q R)-q\left(x_{k} ; Q R\right)\right\|^{2}}{\sigma}\right)
\end{array}
$$

where $\hat{I}(x)$ is the estimated smoothed intensity, $N(x ; S R)$ is a search window of diameter $S R$ around point $x, w_{k}(x)$ is a weight factor, $q(x ; Q R)$ is a patch of diameter $Q R$ centered at $x$ and $\sigma$ is a smoothing parameter ${ }^{2}$. In our experiments, a patch size of $12 \times 12$ was used, with a search window of $50 \times 50 . \sigma$ was chosen by running the NL-means algorithm for 55 different $\sigma$ values for smoothing, from the set $\{1: 1: 10,20: 20: 640,650: 50: 1200\}$. The optimal $\sigma$ values were computed using the following criteria: $C C(D, R), M I(D, R), \eta_{1}(D, R), \eta_{2}(D, R)$; sum of localized versions of all above measures on a $12 \times 12$ window; $h, P$ and $K$ using two-sample KS tests on non-overlapping $12 \times 12$ patches; and $h_{n}, P_{n}$ and $K_{n}$ values computed using KS-test between the residual and the true noise samples (which we know here as these are synthetic experiments). All information theoretic quantities were computed using 40 bins as the image size was $256 \times 256$ (the optimal number of bins for $n$ samples is $O\left(n^{1 / 3}\right)$ ).

The total variation (TV) filter is obtained by minimizing the following energy:

$$
E(I)=\int_{\Omega}|\nabla I(x)| d x
$$

for an image defined on the domain $\Omega$, which gives a geometric heat equation PDE that is iterated some $T$ times starting from the given noisy image as an initial condition. The stopping time $T$ is the equivalent of the smoothing parameter here. For the TV filter, in addition to all the criteria mentioned before, we also tested Gilboa's method

\footnotetext{
${ }^{2}$ Note that we use $\sigma$ to denote the smoothing parameter of the filtering algorithm and $\sigma_{n}^{2}$ to denote the variance of the noise.
}

[11] (assuming knowledge of the noise distribution) and Vanhamel's method [21].

\subsection{Validation method}

In order to validate the $\sigma$ or $T$ estimates produced by these criteria, it is important to see how well they are in tune with those filter parameter values that are optimal with regard to different well-known quality measures between the denoised image and the original image. The most commonly used quality measure is the MSE. However as documented beautifully in [23], MSE has several limitations. Hence we also experimented with structured similarity index (SSIM) developed in [22]; with the L1 difference between the denoised and the original image; and with the $\mathrm{CC}, \mathrm{MI}, \eta_{1}$ and $\eta_{2}$ values between the denoised and the original image (as well as with the sum of their local versions).

\subsection{Results on NL-Means}

Results on NL-Means for Gaussian noise of six different variances are shown in Tables 1 through to 6. In all these tables, $\Delta_{X}=$ absolute difference between ' $X$ ' values as predicted by the criterion, and the optimal ' $X$ ' value. The ' $X$ ' value is defined to be the quality measure ' $X$ ' between the denoised and the true image, chosen here to be $L_{1}$ or the SSIM. $d \sigma_{X}$ is the absolute difference between the $\sigma$ value for NL-means predicted by the criterion and the $\sigma$ value when the quality measure $X$ was optimal. The other quality measures are not shown here to save space. The last two rows of the tables indicate the minimum and maximum of the optimal quality measure values across all the 13 images on which the experiments were run (which gives an idea about the range of the optimal $\Delta_{X}$ values).

Some results on the 'stream' and 'mandrill' images are shown in Figure 2 and 3 with the corresponding residuals. Experiments were also performed on images degraded with bounded uniform noise of total width $2 \times 5 \times 10^{-4}$ and $2 \times 5 \times 10^{-3}$ (on an intensity range of $[0,1]$ ) with results shown in Tables 7 and 8. Similar experiments were run with negative exponential noise where these criteria performed well. The results are not shown here due to insufficient space.

For low and moderate noise levels, it was observed that the criteria $P$ or $K$ produced errors an order of magnitude better than MI, $\eta_{1}$ and $\eta_{2}$ (which were the closest competitors) and even two orders of magnitude better than CC. Our observation was that $\mathrm{CC}$ and information theoretic criteria tend to cause undersmoothing for NL-means. At high noise levels, we saw that all criteria produced a high error in prediction of the optimal parameter. An explanation for this is that the NL-means algorithm by itself does not produce very 
good results at high noise levels, and requires high $\sigma$ values which produce highly structured residuals. For low $\sigma$ values, it produces residuals that resemble noise in the sense of various criteria, but this leads to hugely undersmoothed estimates.

An interesting phenomenon we observed was that the same KS-test based measures (i.e. $P_{n}$ and $K_{n}$ ) between the residuals and the actual noise samples (which we know, as these are synthetic experiments) often did not perform as well as the KS-test measures (i.e. $P$ and $K$ ) between pairs of patches from the residual. We conjecture that this is owing to biases inherent in the NL-means algorithm (as in many others - see [5]) due to which the residuals have different means and variances as compared to the actual noise, even though the residuals may be homogenous. We checked experimentally that the variance of the residuals produced by NL-means under $\sigma$ values optimal in an L1-sense was significantly different from the noise variance.

\subsection{Effect of patch size on the KS test}

The KS test employed here operates on image patches. The patch-size can be a crucial parameter: too low a patch size (say $2 \times 2$ ) will lead to reduction in the discriminatory power of the KS test for this application and cause (false) rejection for all filter parameters, whereas too high a patch size will lead to (false) acceptance for all filter parameters. We chose a patch size so that the number of samples for estimation of the cumulative was sufficient. This was determined in such a way that the maximum absolute error between the estimated and true underlying CDFs was no more than 0.1 with a probability of 0.9 . Then, using the Dvoretzky-Kiefer-Wolfowitz inequality, it follows that there should be at least 149 samples [9]. Hence we chose a patchsize of $12 \times 12$. However, we also performed an experiment with NL-means where the KS-test was performed across multiple scales from 12 to 60 in steps of 8 (for an image of size $256 \times 256$ ), and average $h, P$ and $K$ values were calculated. However for the several experiments described in the previous sections, we just used the patchsize of $12 \times 12$, as the multiscale measure did not produce significantly better results.

\subsection{Results on Total Variation}

Results for total variation diffusion with Gaussian noise of variance $5 \times 10^{-4}$ and $5 \times 10^{-3}$ are shown in Tables 9 and 10. For this method, the KS-based measures performed well in terms of errors in predicting the correct number of iterations and the correct quality measures, but not as well as MI within the restricted stopping time range. The results were also compared to those obtained from Gilboa's method [11] which performed the best, though we would like to remind
Table 1. (NLMeans)Gauss. noise $\sigma_{n}^{2}=0.0001$.

\begin{tabular}{|l|l|l|l|l|}
\hline- & $\Delta_{L 1}$ & $d \sigma_{L 1}$ & $\Delta_{S S I M}$ & $d \sigma_{S S I M}$ \\
\hline$h$ & 0.088 & 10.462 & $\mathbf{0 . 0 0 2}$ & $\mathbf{7 . 6 9 2}$ \\
\hline$P$ & $\mathbf{0 . 0 3 1}$ & $\mathbf{7 . 5 3 8}$ & 0.005 & 12.462 \\
\hline$K$ & 0.040 & 7.846 & 0.004 & 10.308 \\
\hline$C C$ & 0.085 & 9.846 & 0.010 & 17.846 \\
\hline$M I$ & 0.189 & 17.077 & 0.011 & 18.615 \\
\hline$\eta_{1}$ & 0.189 & 17.077 & 0.011 & 18.615 \\
\hline$\eta_{2}$ & 0.176 & 18 & 0.011 & 20.769 \\
\hline Local MI & 0.055 & 8.769 & 0.007 & 16.154 \\
\hline$h_{N M}$ & 0.851 & 31.462 & 0.009 & 16.385 \\
\hline$P_{N M}$ & 0.087 & 13.231 & 0.002 & 4.923 \\
\hline$K_{N M}$ & 0.215 & 19.385 & 0.001 & 5.538 \\
\hline Min & 2.225 & - & 0.888 & - \\
\hline Max & 9.147 & - & 0.986 & - \\
\hline
\end{tabular}

Table 2. (NLMeans)Gauss. noise $\sigma_{n}^{2}=0.0005$.

\begin{tabular}{|l|l|l|l|l|}
\hline- & $\Delta_{L 1}$ & $d \sigma_{L 1}$ & $\Delta_{S S I M}$ & $d \sigma_{S S I M}$ \\
\hline$h$ & 0.029 & 7.692 & $\mathbf{0 . 0 0 3}$ & $\mathbf{1 2 . 7 6 9}$ \\
\hline$P$ & $\mathbf{0 . 0 1 0}$ & $\mathbf{4 . 6 1 5}$ & 0.006 & 17.385 \\
\hline$K$ & 0.014 & 5.385 & 0.004 & 15.077 \\
\hline$C C$ & 0.068 & 8.462 & 0.007 & 15.077 \\
\hline$M I$ & 0.087 & 14.615 & 0.009 & 19.692 \\
\hline$\eta_{1}$ & 0.087 & 14.615 & 0.009 & 19.692 \\
\hline$\eta_{2}$ & 0.232 & 19.923 & 0.015 & 26.538 \\
\hline Local MI & 0.155 & 14.615 & 0.005 & 13.538 \\
\hline$h_{n}$ & 0.436 & 28.154 & 0.003 & 8 \\
\hline$P_{n}$ & 0.047 & 10.769 & 0.003 & 9.692 \\
\hline$K_{n}$ & 0.128 & 16.154 & 0.001 & 5.846 \\
\hline Min & 2.683 & - & 0.884 & - \\
\hline Max & 9.383 & - & 0.981 & - \\
\hline
\end{tabular}

Table 3. (NLMeans)Gauss. noise $\sigma_{n}^{2}=0.001$.

\begin{tabular}{|l|l|l|l|l|}
\hline- & $\Delta_{L 1}$ & $d \sigma_{L 1}$ & $\Delta_{S S I M}$ & $d \sigma_{S S I M}$ \\
\hline$h$ & 0.041 & 9.231 & 0.004 & 16.154 \\
\hline$P$ & $\mathbf{0 . 0 2 4}$ & $\mathbf{5 . 3 8 5}$ & 0.005 & 16.923 \\
\hline$K$ & 0.035 & 7.692 & $\mathbf{0 . 0 0 4}$ & 14.615 \\
\hline$C C$ & 0.151 & 16.154 & 0.004 & $\mathbf{1 2 . 3 0 8}$ \\
\hline$M I$ & 0.126 & 19.231 & 0.008 & 18.462 \\
\hline$\eta_{1}$ & 0.126 & 19.231 & 0.008 & 18.462 \\
\hline$\eta_{2}$ & 0.157 & 26.923 & 0.013 & 29.231 \\
\hline Local MI & 0.191 & 20.308 & 0.003 & 10.308 \\
\hline$h_{n}$ & 0.218 & 22.308 & 0.001 & 6.154 \\
\hline$P_{n}$ & 0.041 & 10 & 0.002 & 12.308 \\
\hline$K_{n}$ & 0.100 & 15.385 & 0.001 & 6.923 \\
\hline Min & 3.069 & - & 0.879 & - \\
\hline Max & 9.601 & - & 0.976 & - \\
\hline & & & & \\
\hline
\end{tabular}


Table 4. (NLMeans)Gauss. noise $\sigma_{n}^{2}=0.005$.

\begin{tabular}{|l|l|l|l|l|}
\hline- & $\Delta_{L 1}$ & $d \sigma_{L 1}$ & $\Delta_{S S I M}$ & $d \sigma_{S S I M}$ \\
\hline$h$ & $\mathbf{0 . 2 0 6}$ & $\mathbf{3 3 . 8 4 6}$ & $\mathbf{0 . 0 0 3}$ & $\mathbf{2 4 . 6 1 5}$ \\
\hline$P$ & 0.207 & 33.846 & 0.003 & 24.615 \\
\hline$K$ & 0.488 & 43 & 0.005 & 33.769 \\
\hline$C C$ & 2.253 & 92.308 & 0.034 & 55.385 \\
\hline$M I$ & 2.677 & 79.538 & 0.054 & 67.231 \\
\hline$\eta_{1}$ & 2.720 & 81.077 & 0.054 & 68.769 \\
\hline$\eta_{2}$ & 2.119 & 105.231 & 0.053 & 105.231 \\
\hline Local MI & 3.889 & 107.846 & 0.069 & 74 \\
\hline$h_{n}$ & 1.337 & 38 & 0.032 & 38 \\
\hline$P_{n}$ & 1.335 & 33.538 & 0.033 & 36.615 \\
\hline$K_{n}$ & 1.336 & 33.538 & 0.034 & 42.769 \\
\hline Min & 4.838 & - & 0.791 & - \\
\hline Max & 10.695 & - & 0.955 & - \\
\hline
\end{tabular}

Table 5. (NLMeans) Gauss. noise $\sigma_{n}^{2}=0.01$.

\begin{tabular}{|l|l|l|l|l|}
\hline- & $\Delta_{L 1}$ & $d \sigma_{L 1}$ & $\Delta_{S S I M}$ & $d \sigma_{S S I M}$ \\
\hline$h$ & 12.121 & 226.692 & 0.202 & 177.462 \\
\hline$P$ & 12.121 & 226.692 & 0.202 & 177.462 \\
\hline$K$ & 12.121 & 226.692 & 0.202 & 177.462 \\
\hline$C C$ & 8.886 & 207.154 & 0.149 & 157.923 \\
\hline$M I$ & 11.701 & 224.462 & 0.195 & 175.231 \\
\hline$\eta_{1}$ & 11.701 & 224.462 & 0.195 & 175.231 \\
\hline$\eta_{2}$ & $\mathbf{6 . 2 1 8}$ & $\mathbf{2 0 0}$ & $\mathbf{0 . 1 1 9}$ & $\mathbf{1 6 3 . 0 7 7}$ \\
\hline Local MI & 12.121 & 226.692 & 0.202 & 177.462 \\
\hline$h_{n}$ & 1.891 & 60.923 & 0.045 & 64 \\
\hline$P_{n}$ & 3.642 & 86.462 & 0.081 & 108 \\
\hline$K_{n}$ & 3.649 & 88 & 0.082 & 115.692 \\
\hline Min & 6.285 & - & 0.735 & - \\
\hline Max & 11.661 & - & 0.933 & - \\
\hline
\end{tabular}

Table 6. (NLMeans)Gauss. noise $\sigma_{n}^{2}=0.05$.

\begin{tabular}{|l|l|l|l|l|}
\hline- & $\Delta_{L 1}$ & $d \sigma_{L 1}$ & $\Delta_{S S I M}$ & $d \sigma_{S S I M}$ \\
\hline$h$ & 14.704 & 906.154 & 0.183 & 643.846 \\
\hline$P$ & 11.290 & 838.462 & 0.140 & 576.154 \\
\hline$K$ & $\mathbf{9 . 5 9 7}$ & $\mathbf{8 0 5 . 3 8 5}$ & $\mathbf{0 . 1 1 8}$ & $\mathbf{5 4 3 . 0 7 7}$ \\
\hline$C C$ & 18.959 & 1020 & 0.249 & 757.692 \\
\hline$M I$ & 19.435 & 1026.154 & 0.253 & 763.846 \\
\hline$\eta_{1}$ & 19.550 & 1027.692 & 0.255 & 765.385 \\
\hline$\eta_{2}$ & 19.435 & 1026.154 & 0.253 & 763.846 \\
\hline Local MI & 19.783 & 1030.769 & 0.258 & 768.462 \\
\hline$h_{n}$ & 24.516 & 1028.462 & 0.305 & 796.923 \\
\hline$P_{n}$ & 26.721 & 1120.769 & 0.325 & 858.462 \\
\hline$K_{n}$ & 26.721 & 1120.769 & 0.325 & 858.462 \\
\hline Min & 11.748 & - & 0.555 & - \\
\hline Max & 17.478 & - & 0.806 & - \\
\hline
\end{tabular}

Table 7. (NLMeans) Unif. noise width $=0.001$.

\begin{tabular}{|l|l|l|l|l|}
\hline- & $\Delta_{L 1}$ & $d \sigma_{L 1}$ & $\Delta_{S S I M}$ & $d \sigma_{S S I M}$ \\
\hline$h$ & 0.055 & 9.692 & $\mathbf{0 . 0 0 3}$ & $\mathbf{1 0 . 9 2 3}$ \\
\hline$P$ & $\mathbf{0 . 0 1 3}$ & $\mathbf{5 . 6 9 2}$ & 0.006 & 14.923 \\
\hline$K$ & 0.021 & 6.615 & 0.005 & 14.000 \\
\hline CC & 0.087 & 11.077 & 0.009 & 17.231 \\
\hline MI & 0.188 & 13.385 & 0.007 & 17.077 \\
\hline$\eta_{1}$ & 0.188 & 13.385 & 0.007 & 17.077 \\
\hline$\eta_{2}$ & 0.267 & 16.846 & 0.009 & 19.000 \\
\hline Local MI & 0.244 & 17.077 & 0.011 & 21.692 \\
\hline$h_{n}$ & 0.770 & 30.231 & 0.008 & 14.538 \\
\hline$P_{n}$ & 0.054 & 10.308 & 0.003 & 7.231 \\
\hline$K_{n}$ & 0.114 & 14.462 & 0.001 & 4.615 \\
\hline Min & 2.339 & -0.887 & -1.195 & - \\
\hline Max & 9.176 & -0.985 & -2.011 & - \\
\hline
\end{tabular}

Table 8. (NLMeans)Unif. noise width $=\mathbf{0 . 0 1}$.

\begin{tabular}{|l|l|l|l|l|}
\hline- & $\Delta_{L 1}$ & $d \sigma_{L 1}$ & $\Delta_{S S I M}$ & $d \sigma_{S S I M}$ \\
\hline$h$ & 0.034 & 10.769 & 0.005 & 18.462 \\
\hline$P$ & $\mathbf{0 . 0 2 7}$ & $\mathbf{9 . 2 3 1}$ & 0.005 & 20.000 \\
\hline$K$ & 0.042 & 12.308 & 0.005 & 16.923 \\
\hline CC & 0.430 & 34.615 & $\mathbf{0 . 0 0 4}$ & $\mathbf{1 4 . 6 1 5}$ \\
\hline MI & 0.137 & 16.923 & 0.007 & 27.692 \\
\hline$\eta_{1}$ & 0.137 & 16.923 & 0.007 & 27.692 \\
\hline$\eta_{2}$ & 0.125 & 21.538 & 0.011 & 35.385 \\
\hline Local MI & 0.477 & 36.308 & 0.004 & 16.308 \\
\hline$h_{n}$ & 0.025 & 9.231 & 0.004 & 20.000 \\
\hline$P_{n}$ & 0.020 & 9.231 & 0.006 & 26.154 \\
\hline$K_{n}$ & 0.025 & 10.769 & 0.006 & 27.692 \\
\hline Min & 3.522 & -0.860 & -1.148 & - \\
\hline Max & 9.835 & -0.970 & -1.834 & - \\
\hline
\end{tabular}

Table 9. (TV)Gauss. noise $\sigma_{n}^{2}=0.0005$.

\begin{tabular}{|l|l|l|l|l|}
\hline- & $\Delta_{L 1}$ & $d t_{L 1}$ & $\Delta_{\text {SSIM }}$ & $d t_{\text {SSIM }}$ \\
\hline$h$ & 0.558 & 53.462 & 0.006 & 56.538 \\
\hline$P$ & 0.522 & 48.462 & 0.006 & 52.308 \\
\hline$K$ & $\mathbf{0 . 5 1 3}$ & $\mathbf{4 6 . 5 3 8}$ & $\mathbf{0 . 0 0 6}$ & $\mathbf{5 0 . 3 8 5}$ \\
\hline CC & 3.487 & 365.000 & 0.088 & 371.923 \\
\hline MI & 0.103 & 20.769 & 0.001 & 23.077 \\
\hline$\eta_{1}$ & 0.103 & 20.769 & 0.001 & 23.077 \\
\hline$\eta_{2}$ & 2.478 & 267.692 & 0.062 & 274.615 \\
\hline Local MI & 0.479 & 36.923 & 0.005 & 32.308 \\
\hline$h_{n}$ & 0.538 & 69.615 & 0.007 & 76.538 \\
\hline$P_{n}$ & 0.523 & 68.846 & 0.007 & 75.769 \\
\hline$K_{n}$ & 0.528 & 69.615 & 0.007 & 76.538 \\
\hline Gilboa [11] & 0.050 & 10.231 & 0.001 & 16.385 \\
\hline VanHamel $[21]$ & 0.818 & 51.954 & 0.009 & 45.031 \\
\hline Min & 2.622 & - & 0.975 & - \\
\hline Max & 4.426 & - & 0.995 & - \\
\hline
\end{tabular}




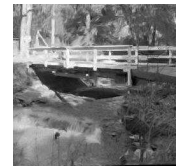

(a)

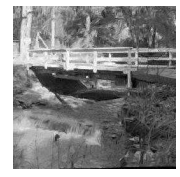

(e)

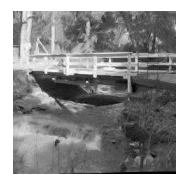

(i)

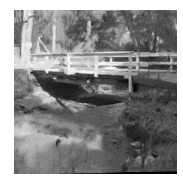

(m)

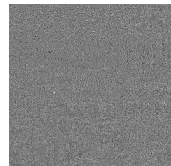

(b)

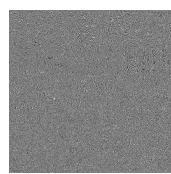

(f)

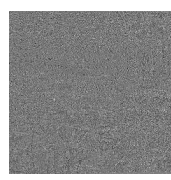

(j)

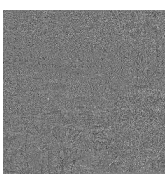

(n)

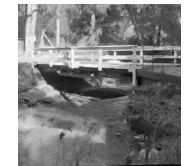

(c)

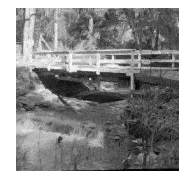

(g)

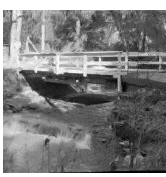

(k)

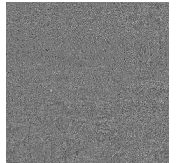

(d)

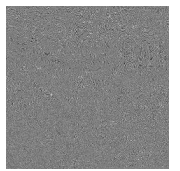

(h)

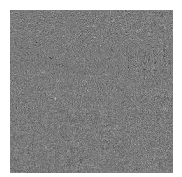

(1)

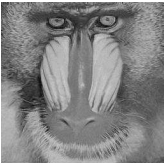

(a)

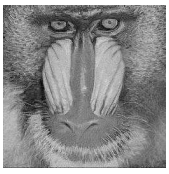

(e)

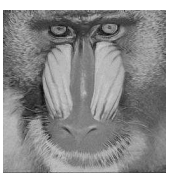

(i)

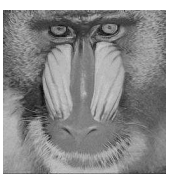

(m)

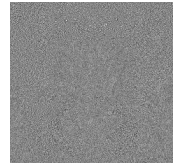

(b)

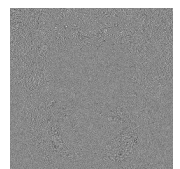

(f)

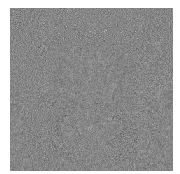

(j)

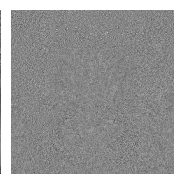

(n)

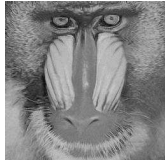

(c)

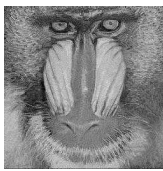

(g)

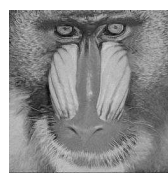

(k)

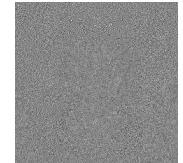

(d)

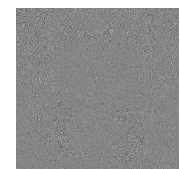

(h)

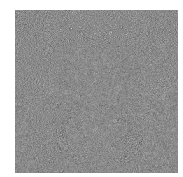

(1)
Figure 2. Images with Gaussian noise with $\sigma_{n}^{2}=5 \times 10^{-3}$ denoised by NLMeans. Parameter selected for optimal noiseness measures: (a): $P$, (c) $K$, (e) $C C$, (g) Ml; and optimal quality measures: (i) $L 1$, (k) SSIM, (m) MI between denoised image and residual. Corresponding residuals in (b),(d),(f),(h),(j),(l),(n). ZOOM IN PDF FOR A BETTER VIEW.

the reader that Gilboa's method requires knowledge of the full noise distribution. Also, in the case of total variation, the KS-based measures did not outperform MI. An explanation for this is that the total variation method is unable to produce homogenous residuals for its optimal parameter set, as it is specifically tuned for piecewise constant images. This assumption does not hold good for commonly occurring natural images. As against this, NL-means is a filter expressly derived from the assumption that patches in 'clean' natural images (and those with low or moderate noise) have several similar patches in distant parts of the image.

\section{Discussion and Avenues for Future Work}

In this paper, we have contributed to and critiqued independence-based criteria for filter parameter selection and presented a criterion that measures the homogeneity of the residual statistics. On the whole, we have contributed to the paradigm of exploiting statistical properties of the residual images for driving the denoising algorithm. The proposed noiseness measures require no other assumptions except that (1) the noise should be i.i.d. and additive, and that (2) a loose lower bound on the noise variance is known to prevent false minima with extreme undersmoothing. Unlike CC or MI, the KS-based noiseness measures are guaranteed not to eyield false minima in case of oversmoothing.

The KS-based noiseness criteria require averaging of the $P$ or $K$ values from different patches. For future work, this can be replaced by performing $k$-sample versions of the Kolmogorov-Smirnov tests or related tests such as Cramer von-Mises [12] between individual patches versus a pooled sample containing the entire residual image. This will produce a single $K$ or $P$ value for the whole image.

The assumption of i.i.d. noise may not hold in some denoising scenarios. In case of a zero-mean Gaussian noise model with intensity dependent variances, a heuristic approach would be to normalize the residuals suitably using feedback from the denoised intensity values (regarding them as the 'true' image values) and then running the KStests. The efficacy of this approach needs to be tested on 
Table 10. (TV)Gauss. noise $\sigma_{n}^{2}=0.005$.

\begin{tabular}{|l|l|l|l|l|}
\hline- & $\Delta_{L 1}$ & $d t_{L 1}$ & $\Delta_{S S I M}$ & $d_{\text {SSIM }}$ \\
\hline$h$ & 0.665 & 129.615 & 0.008 & 102.692 \\
\hline$P$ & 0.493 & 109.615 & 0.006 & 80.385 \\
\hline$K$ & 0.430 & 102.308 & $\mathbf{0 . 0 0 6}$ & $\mathbf{7 3 . 0 7 7}$ \\
\hline CC & 2.156 & 350.769 & 0.073 & 376.923 \\
\hline MI & 0.422 & 88.846 & 0.012 & 118.077 \\
\hline$\eta_{1}$ & $\mathbf{0 . 4 2 2}$ & $\mathbf{8 8 . 8 4 6}$ & 0.012 & 118.077 \\
\hline$\eta_{2}$ & 1.849 & 296.923 & 0.063 & 331.538 \\
\hline Local MI & 5.475 & 270.769 & 0.084 & 240.769 \\
\hline$h_{n}$ & 0.194 & 59.615 & 0.008 & 96.538 \\
\hline$P_{n}$ & 0.221 & 74.231 & 0.008 & 115.000 \\
\hline$K_{n}$ & 0.216 & 75.769 & 0.008 & 116.538 \\
\hline Gilboa $[11]$ & 0.094 & 60.000 & 0.002 & 42.000 \\
\hline VanHamel $[21]$ & 4.658 & 303.400 & 0.068 & 262.631 \\
\hline Min & 4.995 & - & 0.892 & - \\
\hline Max & 11.284 & - & 0.980 & - \\
\hline
\end{tabular}

denoising algorithms that are capable of handling intensity dependent noise. In case the noise obeys a Poisson distribution (for which the variance is equal to the mean, and hence which is neither fully additive nor multiplicative), there are two ways to proceed: either apply a variance stabilizer transformation which converts the data into that corrupted by Gaussian noise with variance of one, or else suitably change the definition of the residual itself.

Moreover, the existence of a universally optimal parameter selector is not yet established: different criteria may perform better or worse for different denoising algorithms or with different assumptions on the noise model. This is, as per our survey of the literature, an open problem in image processing. Lastly, despite encouraging experimental results, there is no established theoretical relationship between the performance of noiseness criteria for filter parameter selection and the 'ideal' parameters in terms of image quality criteria like MSE. A detailed study of risk-based criteria such as those in [7] may be important in this context.

\section{References}

[1] http://www.stanford.edu/ slansel/ DenoiseLab/download.htm.

[2] J.-F. Aujol, G. Gilboa, T. Chan, and S. Osher. Structuretexture image decomposition-modeling, algorithms and parameter selection. Int. J. Comput. Vision, 67(1):111-136, 2006.

[3] D. Brunet, E. Vrscay, and Z. Wang. The use of residuals in image denoising. In ICIAR, pages 1-12, 2009.

[4] A. Buades, B. Coll, and J.-M. Morel. A review of image denoising algorithms, with a new one. Multiscale modelling and simulation, 4(2):490-530, 2005.
[5] P. Chatterjee and P. Milanfar. Is denoising dead? Accepted to IEEE Trans. on Image Processing, 2010.

[6] T. Cover and J. Thomas. Elements of Information Theory. Wiley-Interscience, New York, USA, 1991.

[7] D. Donoho and I. Johnstone. Ideal adaptation via wavelet shrinkage. Biometrika, 81:425-455, 1994.

[8] D. Donoho and I. Johnstone. Adapting to unknown smoothness via wavelet shrinkage. Journal of the American Statistical Association, 90(432):1200-1224, 1995.

[9] A. Dvoretzky, J. Kiefer, and J. Wolfowitz. Asymptotic minimax character of the sample distribution function and of the classical multinomial estimator. Annals of Mathematical Statistics, 27(3):642-669, 1956.

[10] M. Elad and M. Aharon. Image denoising via learned dictionaries and sparse representation. In $C V P R$, pages 1-6, 2006.

[11] G. Gilboa, N. Sochen, and Y. Zeevi. Estimation of optimal PDE-based denoising in the SNR sense. Image Processing, IEEE Transactions on, 15(8):2269-2280, Aug. 2006.

[12] J. Kiefer. K-sample analogues of the Kolmogorov-Smirnov and Cramer-Von Mises tests. Annals of Mathematical Statistics, 30(2):420-447, 1959.

[13] D. Martin, C. Fowlkes, D. Tal, and J. Malik. A database of human segmented natural images and its application to evaluating segmentation algorithms and measuring ecological statistics. In ICCV, volume 2, pages 416-423, 2001.

[14] P. Mrázek and M. Navara. Selection of optimal stopping time for nonlinear diffusion filtering. Int. J. Comput. Vision, 52(2-3):189-203, 2003.

[15] P. Perona and J. Malik. Scale-space and edge detection using anisotropic diffusion. IEEE Transactions on Pattern Analysis and Machine Intelligence, 12(7):629-639, 1990.

[16] J. Polzehl and V. Spokoiny. Image denoising: a pointwise adaptive approach. Annals of Statistics, 31(1):30-57, 2003.

[17] A. Rajwade, A. Banerjee, and A. Rangarajan. Image filtering driven by level curves. In EMMCVPR, pages 359-372, 2009.

[18] L. Rudin and S. Osher. Total variation based image resoration with free local constraints. In International Conference on Image Processing, pages 31-35, 1994.

[19] Ö. Subakan, B. Jian, B. Vemuri, and E. Vallejos. Feature preserving image smoothing using a continuous mixture of tensors. In $I C C V$, pages 1-6, 2007.

[20] H. Takeda, S. Farsiu, and P. Milanfar. Kernel regression for image processing and reconstruction. IEEE Transactions on Image Processing, 16(2):349-366, 2007.

[21] I. Vanhamel, C. Mihai, H. Sahli, A. Katartzis, and I. Pratikakis. Scale selection for compact scale-space representation of vector-valued images. Int. J. Comput. Vision, 84(2):194-204, 2009.

[22] Z. Wang and A. Bovik. A universal image quality index. IEEE Signal Processing Letters, 9(3):81-84, 2002.

[23] Z. Wang and A. Bovik. Mean squared error: love it or leave it? - a new look at signal fidelity measures. IEEE Signal Processing Magazine, 26(1):98-117, 2009.

[24] J. Weickert. Coherence-enhancing diffusion of colour images. Image and Vision Computing, 17:201212, 1999. 